\title{
EFFECTS OF MEMBRANE STIFFENING ON FOCAL-ADHESION BONDING UNDER STEADY AND UNSTEADY CONDITIONS
}

\author{
I. Avrahami, M. Gharib; \\ California Institute of Technology, Pasadena, CA.
}

Platelets adhesion occurs at focal adhesions (FA), where cell-membrane receptors bind specifically to substrate proteins and couple to each other and to the cytoskeleton via various cellular proteins. Some of the reactions that follow the ligand-receptor binding at the FA may affect mechanical determinants of the cell-substrate attachment. The resulting molecular structure suggests that the cortex stiffens at the FA, which likely affects platelet adhesion. The present work explores that hypothesis using a numerical simulation of the 3D membrane flexible structure under steady and unsteady bond kinetic and detachment forces. The cortex is modeled as a shell anchored to the substrate by unevenly distributed adhesion forces and subjected to externally detachment forces. In the simulated models, the steady case address to a stabilized condition, when both the adhesion forces and the detachment forces are steady. In the unsteady case, however, some aspects of bond kinetics and unsteadiness in the external detachment forces are incorporated. The commercial finite-element package ADINA (Watertown, MA) is used to solve the 3D time-dependent structural equations in the model. The results show the effect of cortex stiffening at the focal adhesion sites on the membrane deformation for both the steady and unsteady cases. The consequent internal stresses are described and the effects of membrane stiffening on the bonding forces are compared for the different cases. In addition, the effect of uneven distribution of focal adhesion complexes on the cells' structural support is evaluated and the consequences on cell function and behaviors are discussed. 